\title{
Age-dating Luminous Red Galaxies observed with the Southern African Large Telescope
}

\author{
Ando Ratsimbazafy*1, Catherine Cress ${ }^{2,3}$, Steve Crawford ${ }^{4}$, Mathew Smith ${ }^{5}$ \\ ${ }^{1}$ Centre for Space Research, North-West University, Potchefstroom 2520, SA \\ ${ }^{2}$ Physics Department, University of the Western Cape, Private bag X17, Cape Town 7535, SA \\ ${ }^{3}$ Centre of High Performance Computing, 15 Lower Hope St, Rosebank, Cape Town 7700, SA \\ ${ }^{4}$ South African Astronomical Observatory, PO Box 9 Observatory, Cape Town 7935, SA \\ ${ }^{5}$ School of Physics and Astronomy, University of Southampton, Southampton SO17 1BJ, UK \\ E-mail: ando.ratsimbazafy@nwu.ac.za, ccress@csir.co.za, crawford@saao.ac.za, \\ mat.smith@soton.ac.uk
}

\begin{abstract}
There have been a number of attempts to measure the expansion rate of the Universe using agedating of Luminous Red Galaxies (LRGs). Assuming that stars in LRGs form at the same time, age-dating of two populations of LRGs at different redshifts can provide an estimate of the time difference associated with the corresponding redshift interval $(d z / d t)$. This gives a direct estimate of the Hubble parameter $H(z)$ at the average redshift of the two populations. We explore the validity of this method by using two different sets of data. Firstly, we measure a value of the Hubble parameter $H(z)$ at a redshift of $z \sim 0.47$. Our measurement is based on analysis of high quality spectra of LRGs obtained with the Southern African Large Telescope (SALT) in two narrow redshift ranges of $z \sim 0.40$ and $z \sim 0.55$ as part of an initial pilot study. Ages were estimated from fitting single stellar population models to the full observed spectra. The actual measurement presents the best estimate of $H(z)$ via this method at $z \sim 0.5$ to date. Secondly, we select a homogeneous sample of passively evolving galaxies over $0.20<z<0.40$ from the Sloan Digital Sky Survey Data Release Seven (SDSS DR7) catalogue by applying a refined criteria, which is based on absolute magnitude. Three additional $H(z)$ measurements were obtained at $z \sim$ $0.28, z \sim 0.30$ and $z \sim 0.32$. We present the $H(z)$ estimates and their cosmological constraints using the two different datasets.
\end{abstract}

SALT Science Conference 2015 -SSC2015-

1-5 June, 2015

Stellenbosch Institute of Advanced Study, South Africa

*Speaker. 


\section{Introduction}

The expansion rate of the Universe, traced by the Hubble parameter $H(z)$, can potentially be measured by the differential evolution of massive and passive elliptical galaxies such as Luminous Red Galaxies (LRGs). This technique is well known as Cosmic Chronometers (CC), and was originally proposed by [1]. The determination of the Hubble parameter is one of the most important cosmological measurements since it depends on the energy components of the Universe (e.g. dark energy). It is therefore crucial to precisely measure $H(z)$ in order to reveal the nature of the energy components of the Universe.

Most cosmological probes only measure the expansion rate integrated along a line-of-sight (e.g. from the standard candels - Supernovae type Ia, from the standard rulers - Baryonic Acoustic Oscillations). However, the CC method provides an independent technique which allows a measurement at a specific redshift without relying on the nature of the metric between the chronometer and us, and this can provide tighter constraints on cosmological parameters. The Hubble parameter formalism from this method is very straightforward:

$$
H(z)=-\frac{1}{(1+z)} \frac{d z}{d t}
$$

$z$ is the cosmological redshift where the measurement is taken and $t$ is the age of the Universe. The term $d z / d t$ can be approximated by determining the time interval $\Delta t$ corresponding to a given $\Delta z$, where $\Delta z$ is centered at redshift $z$. From the Equation 1.1, it is then possible to estimate the expansion rate of the Universe at different epochs using the age differences of LRGs. The overall method is valid when assuming that most stars in LRGs formed near the beginning of the Universe at a similar time, then measuring the age difference between ensembles of LRGs at two different redshifts provides the differential quantity $\Delta z / \Delta t$ required to estimate the Hubble parameter $H(z)$. This differential quantity is defined as the standard clocks. Using this significantly reduces systematics associated with the age determination. Some systematic errors in the absolute age measurements have been a subject of discussion but such errors could be cancelled by using the relative ages of these galaxies. Furthermore, only the galaxy evolution that takes place between the redshifts, where the difference is taken, is the most important while using this method.

A number of scientists have attempted to use this method to track the evolution of $H(z)$ up to redshift $z \sim 2$, and these results have been used to constrain cosmological parameters, for example in $[2,3,4,5,6,7,8]$. In each measurement, the authors assumed that LRGs are massive, passively-evolving elliptical galaxies that are homogeneous populations forming their stars at high redshift, and they fit single-burst equivalent ages to the galaxies. These galaxies represent the ideal population to trace the differential age evolution of the Universe.

Age-dating of galaxies is an important topic in the galaxy evolution by the creation and the use of population synthesis tools. The modelling of the stellar evolution becomes more and more reliable than earlier generations of models. However there is still a limitation by certain physical issues, the imperfection of the evolutionary tracks, and the incompleteness of the stellar libraries. The principle of the stellar population synthesis method is to match the theoretical features with the observational features of a galaxy. Thus different fitting techniques are used such as the Spectral Energy Distributions (SEDs), spectral line indices or full spectrum fitting. We explored the full spectrum fitting technique. 
Here, we present the use of two different datasets to measure Hubble parameters $H(z)$ and their cosmological constraints. First, we obtained observations of LRGs from the Southern African Large Telescope (SALT) and then used an additional data from the Sloan Digital Sky Survey Data Release Seven (SDSS DR7).

\section{SALT-LRGs}

Not only we explored the optimal set-ups for the similar experiment using the SALT telescope but we also studied the estimation of the uncertainties in the mean age as function of galaxies that could possibly be observed [9]. We then obtained, reduced and analysed 16 long-slit spectra of LRGs recently observed with the SALT telescope. These galaxies were selected from the $2 \mathrm{dF}-$ SDSS LRG and QSO (2SLAQ) and MegaZ-LRGs catalogues at redshift $z \simeq 0.40$ and $z \simeq 0.55$. Our selection was based on stellar mass, brightness and emission lines of a galaxy in order to have a sample of old and massive passively-evolving galaxies. For all observations, the basic reductions were already performed by the semi-automated code PySALT ${ }^{1}[10]$. Further reduction was performed by following the standard long-slit data reduction technique with IRAF ${ }^{2}$. All spectra were corrected for foreground Galactic extinction.

Although in our previous work [9] we did find that LRGs may be better described by slightly extended star formation histories, we only fitted the LRGs to Simple Stellar Populations (SSPs), with the same models, in order to compare this work to previous studies. We used the ULySS ${ }^{3}$ (University of Lyon Spectroscopic analysis Software [11]) package for the fits. ULySS fits the entire spectrum with a model in the form of linear combinations of non-linear components, corrected for the kinematics and multiplied by a polynomial at the same time. The use of multiplicative polynomials makes this method insensitive to the effects of the flux calibration uncertainties and the Galactic extinction.

The SSP models that we used are based on GALAXEV models [12] (BC03) which were generated using the STELIB library with a resolution of about $3 \AA$ FWHM across the whole spectral range 3200 - $9500 \AA$, the Chabrier Initial Mass function (IMF) with a mass of 0.1 to $100 \mathrm{M}_{\odot}$ and a slope of -1.35 and Padova 1994 isochrones. The total of 696 SSP models cover ages from 0.1 to $20 \mathrm{Gyr}$ and $[\mathrm{Fe} / \mathrm{H}]$ from -2.3 to 0.4 dex.

We performed the full spectrum fitting after matching the resolution of both model and observed spectra. The reliability of the fits were also checked carefully. Despite the number of galaxies at $z \simeq 0.55$ (only 6 as compared to 10 at $z \simeq 0.40$ ), we found an age-redshift relation, that is, the mean age at $z \simeq 0.40$ is older $(3.88 \pm 0.20 \mathrm{Gyr})$ than that at $z \simeq 0.55(2.80 \pm 0.18 \mathrm{Gyr})$. We found younger ages than the total sample of red galaxies in [4] at both redshifts but we note that we have a small number of galaxies in our sample. The error on the mean age was obtained by applying the standard error propagation technique.

The mean age of the sample at each redshift was used to measure the differential ages. Applying Equation 1.1 to the intermediate redshift between $z \simeq 0.40$ and $z \simeq 0.55$, we obtained a new

\footnotetext{
${ }^{1}$ pysalt.salt.ac.za

${ }^{2}$ Image Reduction and Analysis Facility, a software system distributed by the National Optical Astronomy Observatories (NOAO). Available at http://iraf.noao.edu/

${ }^{3}$ Available at http://ulyss.univ-lyon $1 . f r /$
} 
observational Hubble parameter $H(z \simeq 0.47)=89 \pm 23 \mathrm{~km} \mathrm{~s}^{-1} \mathrm{Mpc}^{-1}$ using SSP equivalent ages (see Figure 1). The most comparable measurement at the same redshift is by [4], who measured a value of $H(z)=97 \pm 60 \mathrm{~km} \mathrm{~s}^{-1} \mathrm{Mpc}^{-1}$ at $z=0.48$. Our value is consistent with the standard cosmology model with the parameters $\Omega_{m}=0.315$, and $H_{0}=67.3 \mathrm{~km} \mathrm{~s}^{-1} \mathrm{Mpc}^{-1}$.

We have addressed the estimated number of galaxies required to obtain the desired precision in [9]. In reality, we could not reach the estimated accuracy because of the observation constraints such as the quality of the observed spectra in terms of $\mathrm{S} / \mathrm{N}$, or the fact that the number of observed objects could not complete our original estimation. A larger and better quality sample would help us to reach our main goal for the future. The relatively small sample presented here was part of an initial pilot study and further observations will be required to improve on the measurement.

\section{Additional sample: SDSS-LRGs}

From the previous section, we obtained only one measurement of $H(z)$ using SALT-LRGs. This measurement is not enough to investigate the $H(z)$ constraining power on the determination of cosmological parameters, more measurements were needed. We then selected a homogeneous sample of passively evolving elliptical galaxies from the SDSS catalogue over $0.20<z<0.40$. Further selection was applied to it to create a quiescent sample free from any emission lines and a sample which contains massive galaxies. We divided the redshift range into 10 redshift bins with a step of $\delta z=0.02$. As in the previous section, the full spectrum fitting with the same software and models was used to obtain SSP equivalent ages and other parameters.

In order to improve the fits, all galaxy spectra within a redshift bin were combined to obtain a high S/N spectrum. The fitting was performed over the whole wavelength coverage of the co-added spectra, and its reliability was checked (with Monte Carlo simulations, $\chi^{2}$ and convergence maps) before validating the SSP results.

From establishing the age-redshift relation, we adopted the linear fitting procedure to fit ages in a specific redshift bin $t\left(z_{i}\right)$. The slope of the fit was therefore the value of $d z / d t$ which is related to the Hubble parameter at an effective redshift $z_{\text {eff }}=\left(z_{\max }+z_{\min }\right) / 2$, according to the Equation 1.1. Precisely, it is given by the following equation:

$$
H\left(z_{e f f}\right)=-\frac{1}{\left(1+z_{e f f}\right)}\left(\frac{d t}{d z}\right)^{-1}
$$

The difference $\Delta z$ in $z_{\min }$ and $z_{\max }$ should not be great and not be small. After testing different $\Delta z$ (from lowest 0.8 to highest 0.16 ), the optimized $\Delta z$ was set to be 0.14 with which all ages were used and the number of ages were the same. Therefore, 8 age data points were used in each subsample, leading to three values of $H(z)$ at the following effective redshifts $z \simeq 0.32, z \simeq 0.30$ and $z \simeq 0.28$. The corresponding error on the $H(z)$ was obtained by using the error propagation technique. The stacking technique provides us less than $10 \%$ uncertainties in age estimates which leads to better $H(z)$ estimates compared with the values found in the literature.

The $H(z)$ results match the $\Lambda \mathrm{CDM}$ cosmology, see Figure 1 . When testing different models, we found some model dependence. The sensitivity and the different ingredients of models led to some changes on $H(z)$ estimates. This is one of the sources of systematic errors when adopting the $\mathrm{CC}$ technique. A further investigation on this is in progress. The possibility of the contribution of 
the young stellar populations in the relative age determination was also tested. We fitted two stellar components of one young (from $0.1 \mathrm{Gyr}$ to $2 \mathrm{Gyr}$ ) and one old (from $2 \mathrm{Gyr}$ - age of the Universe) stellar populations and found that the fraction of the young population is negligible.

The comparison plot between our measurements and the other observational Hubble parameters $H(z)$ is shown in Figure 1. Datasets from $[3,4,5,6,7,13]$ are included to check the $H(z)$ evolution up to $z \sim 2$. Note that all $H(z)$ shown in this plot are obtained by applying the same technique of $\mathrm{CC}$, except the results from [13] which are from the Baryonic Acoustic Oscillations (BAO). These data points are included in our analysis in order to compare our results on cosmological constraints (see Section 4) with the previous works.

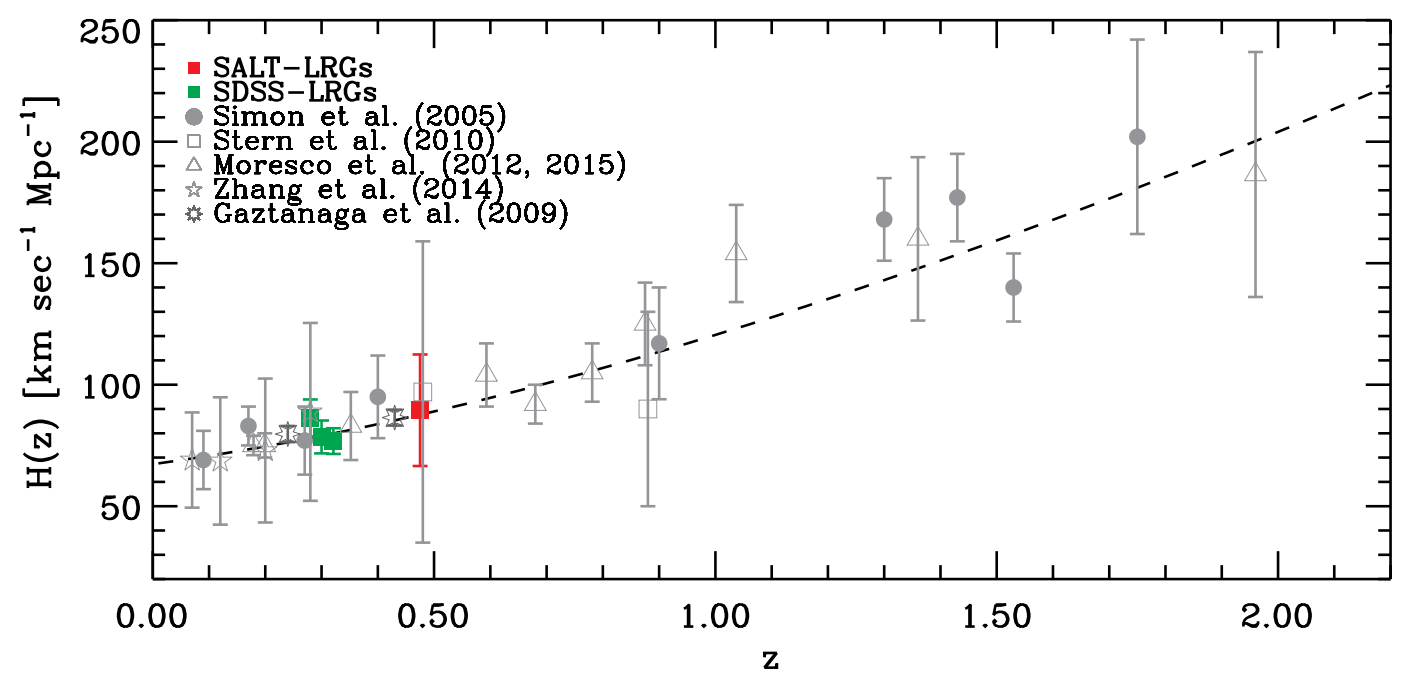

Figure 1: All available observational $H(z)$ data points. The green points are our measurements using SDSSLRGs and the red points for using SALT-LRGs. The dashed line is the theoretical $H(z)$ of a flat $\Lambda$ CDM cosmology model by PLANCK assuming $H_{0}=67.3 \mathrm{~km} \mathrm{~s}^{-1} \mathrm{Mpc}^{-1}$ and $\Omega_{m}=0.315$. For the SDSS-LRGs : $H(z \simeq 0.32)=86.3 \pm 7.6 \mathrm{~km} \mathrm{~s}^{-1} \mathrm{Mpc}^{-1}, H(z \simeq 0.30)=78.5 \pm 6.8 \mathrm{~km} \mathrm{~s}^{-1} \mathrm{Mpc}^{-1}$ and $H(z \simeq 0.28)=76.8 \pm 5.3$ $\mathrm{km} \mathrm{s}^{-1} \mathrm{Mpc}^{-1}$ were estimated when fitting a straight line on the ages over $0.24<z<0.40,0.22<z<0.38$, and $0.20<z<0.36$, respectively. For the SALT-LRGs: $H(z \simeq 0.47)=89 \pm 23 \mathrm{~km} \mathrm{~s}^{-1} \mathrm{Mpc}^{-1}$ was measured using the differential ages.

\section{Cosmological Constraints}

We made use of our $H(z)$ measurements combined with the recent and available $H(z)$ in the literature (all data points plotted in Figure 1) to derive new constraints on the determination of cosmological parameters. We also explored the availability of combining those $H(z)$ data with other cosmological measurements: BAO, Wilkinson Microwave Anisotropy Probe (WMAP) and Hubble Space Telescope (HST) to better determine the constraints on the parameter calculations. The first calculation was done with our own code, the second one was performed by using the publicly available code cosmoMC. Both calculations were based on the Markov Chain Monte Carlo (MCMC) techniques to determine the cosmological parameters in the standard $\Lambda \mathrm{CDM}$ model, such as the 
density of matter parameter $\Omega_{m}$, the cosmological constant $\Omega_{\Lambda}$, the spatial curvature parameter today $\Omega_{k}$ and the Hubble constant today $H_{0}$.

Our new measurements impact current constraints on cosmological parameters even though only 4 data points were added to the old ones ( 27 data points). The 1D marginalized probability distribution of using 31 data points shows a more reduced distribution than using the old datasets, while they tighten each parameter contour of the confidence level in the 2D marginalization plots. The best fit parameters are shown in Table 1 for flat $\Lambda$ CDM model.

We further combined the above observational $H(z)$ measurements datasets with the other major datasets such as WMAP nine year data (WMAP9), BAO and HST to investigate their constraints on the determination of the cosmological parameters. The latest results of the Cosmic Microwave Background (CMB) temperature and polarization power spectra from WMAP9 [14] were used as well as the BAO measurements from:

- 6dF Galaxy Redshift Survey (6dFGRS) at $z=0.106$ [15]

- SDSS-LRGs DR7 sample at $z=0.35$ [16]

- SDSS-LRGs DR9 sample at $z=0.57$ [17]

- WiggleZ survey at $z=0.44, z=0.60$ and $z=0.73$ [18]

The last dataset used was HST measurement of the Hubble parameter today $H_{0}=73.8 \pm 2.3 \mathrm{~km}$ $\mathrm{s}^{-1} \mathrm{Mpc}^{-1}$. This value was obtained from the magnitude-redshift relation of 235 Supernovae type Ia observed with HST.

For a flat $\Lambda \mathrm{CDM}$, we obtained the constraints shown in Table 2 . It shows mainly the effect of adding $H(z)$ data on cosmological parameters by considering four basic data combinations: WMAP9, WMAP9 $+H_{0}$ HST, WMAP9+BAO and WMAP9+BAO $+H_{0}$ HST. Through these results, we can see that the overall joint constraints are consistent with the standard cosmological model. We find that adding the $H(z)$ datasets and $H_{0}$ HST shows a noticeable constraint on $H_{0}, \Omega_{m}$ parameters. This is also due to the better constraint on the current Hubble constant $H_{0}$. WMAP9+Hz joint analysis does not provide any improvement over the WMAP9 alone since the WMAP9 constraint is much more precise than $H(z)$ constraint. The WMAP9+BAO data alone only give very weak constraints on $\Omega_{m}$. However, a combination of WMAP9+BAO with the measurements of Hubble parameter $H(z)$ significantly improves the $1-\sigma$ error on both parameters. The parameter values of $H_{0}=69.4 \pm 1.3 \mathrm{~km} \mathrm{~s}^{-1} \mathrm{Mpc}^{-1}$ and $\Omega_{m}=0.295_{-0.012}^{+0.011}$ are consistent with results of [8], where only 25 data points were used. More external datasets are needed to further investigate on cosmological constraints, and determine different cosmological parameters.

Table 1: Constraints on cosmological parameters from LRG measurements of $H(z)$. Marginalized constraints on $H_{0}, \Omega_{m}$, and $\Omega_{\Lambda}$ at $1-\sigma$ for a flat $\Lambda \mathrm{CDM}$ model

\begin{tabular}{ccc}
\hline \hline Model Parameter & 27 data points & 31 data points \\
\hline$H_{0}$ & $68.9_{-2.5}^{+2.7}$ & $68.6 \pm 2.3$ \\
$\Omega_{m}$ & $0.320_{-0.047}^{+0.047}$ & $0.321_{-0.060}^{+0.048}$ \\
$\Omega_{\Lambda}$ & $0.679_{-0.050}^{+0.047}$ & $0.675_{-0.048}^{+0.060}$ \\
\hline
\end{tabular}


Table 2: Combining LRG results from $H(z)$ with other measurements. Marginalized constraints at 1- $\sigma$ on $H_{0}, \Omega_{m}$ parameters obtained for a flat $\Lambda \mathrm{CDM}$ model. $\mathrm{Hz}$ means including $H(z)$ datasets.

\begin{tabular}{lcc}
\hline \hline & $H_{0}$ & $\Omega_{m}$ \\
\hline WMAP9+Hz & & \\
WMAP9+ $H_{0} \mathrm{HST}+\mathrm{Hz}$ & $71.7_{-2.3}^{+2.6}$ & $0.319_{-0.04}^{+0.048}$ \\
WMAP9+BAO & $68.270_{-0.034}^{+0.043}$ \\
WMAP9+BAO+Hz & $69.4 \pm 1.3$ & $0.299_{-0.016}^{+0.022}$ \\
WMAP9+BAO+ $H_{0} \mathrm{HST}$ & $72.5_{-0.6}^{+0.7}$ & $0.285_{-0.012}^{+0.011}$ \\
WMAP9+BAO+ $H_{0} \mathrm{HST}+\mathrm{Hz}$ & $70.4 \pm 1.1$ & $0.290 \pm 0.010$ \\
\hline
\end{tabular}

\section{Conclusions}

The differential age or cosmic chronometers technique is an interesting way to measure the expansion rate of the Universe, since it gives a direct measurement between two distinct redshifts. It uses massive and passive ellipticals. We have explored this technique using 16 long-slit spectra of LRGs observed with SALT telescope. A massive and passively evolving sample of LRGs at redshift $z \simeq 0.40$ and $z \simeq 0.55$ has been selected. We have reduced and analysed those spectra. The full spectrum fitting with BC03 models has been applied to extract the SSP equivalent ages. The mean age of galaxies in each redshift has been used to estimate $H(z)$ at $z \simeq 0.47$. We have added additional sample of passive galaxies over $0.10<z<0.40$ from SDSS DR7 in order to increase the number of measured $H(z)$. Three additional measurements of $H(z)$ were obtained at $z \simeq 0.28, z \simeq 0.30$ and $z \simeq 0.32$. The four $H(z)$ values combined with external datasets have been employed to constrain the cosmological parameters. We have obtained noticeable constraints and parameter values which are consistent with the standard cosmological model and very consistent with previous results.

Further investigations on cosmological constraints should be done by adding more datasets, for example the new PLANCK data. The systematic errors between SSP models remain a subject of discussion. Further analysis on this will be performed.

\section{Acknowledgments}

ALR thanks funding from National Research Foundation. Some of the observations reported in this paper were obtained with the Southern African Large Telescope (SALT) under program 2011-3-RSA_OTH-026 and 2012-1-RSA_OTH-013 (PI: Ando Ratsimbazafy).

\section{References}

[1] Jimenez R \& Loeb A 2002 ApJ 57337

[2] Jimenez R, Verde L, Treu T, Stern D 2003 ApJ 593622

[3] Simon J, Verde L, Jimenez R 2005 PhRvD 71123001 
[4] Stern D, Jimenez R, Verde L, Kamionkowski M, Stanford S A 2010 JCAP 28

[5] Moresco M, et al. 2012 JCAP 86

[6] Moresco M 2015, MNRAS 45016

[7] Zhang C, et al. 2012, RAA 141221

[8] Zheng W, et al. 2014 International Journal of Modern Physics D 231450051

[9] Crawford S M, Ratsimbazafy A L, Cress C M, et al. 2010 MNRAS 4062569

[10] Crawford S M, Still M, Schellart P, et al. 2010 PROCSPIE 7737773725

[11] Koleva M, Prugniel P, Bouchard A, Wu Y 2009 AEA 5011269

[12] Bruzual G, \& Charlot S 2003 MNRAS 3441000

[13] Gaztañaga E, Cabré A, \& Hui L 2009 MNRAS 3991663

[14] Hinshaw G, Larson D, Komatsu E, et al. 2013 APJS 20819

[15] Beutler F, Blake C, Colless M, et al. 2011 MNRAS 4163017

[16] Padmanabhan N, Xu X, Eisenstein D J, et al. 2012 MNRAS 4272132

[17] Anderson L, Aubourg É, Bailey S, et al. 2014 MNRAS 44124

[18] Blake C, Brough S, Colless M, et al. 2012 MNRAS 425405 